\title{
Relating the deBroglie and Compton Wavelengths to the Velocity of Light?
}

\author{
Gordon R. Kepner ${ }^{1}$ \\ ${ }^{1}$ Membrane Studies Project, USA \\ Correspondence: Gordon R. Kepner, Membrane Studies Project, USA. E-mail: gkepnermsp@gmail.com
}

Received: May 27, 2018

Accepted: July 12, 2018

Online Published: July 26, 2018

doi:10.5539/apr.v10n4p102

URL: https://doi.org/10.5539/apr.v10n4p102

\begin{abstract}
No report in the literature has directly described this relation. New constants for particles are presented. One relates to the Compton wavelength, called here the "mass-wave" constant for all particles. The other relates to the deBroglie wavelength, called here the "velocity-wave" constant for a particle. An equation is derived based on these two constants encapsulating a fundamental relation between the matter-states, particle and wave, to the velocity of light. New approaches to the Uncertainty relations are shown. The basic Schrodinger equation is derived from the perspective of a non-dimensional second-order differential equation free of any assumed empirical constants. The resulting time-dependent wave equation for a free particle was then expressed in terms of the particle velocity and deBroglie wavelength.
\end{abstract}

Keywords: deBroglie particle constant, matter waves, new physical constants, new fundamental equation, wavestate, particle-state

\section{Introduction}

There appears to be no study in the literature that has directly examined this relation. One possible reason is that certain constants relevant to the relation have generally gone unrecognized. This paper describes a constant dependent on the Compton wavelength (Compton, 1923) and another dependent on the deBroglie wavelength (deBroglie, 1925) The analysis relates these constants to the velocity of light, the Uncertainty relation (Heisenberg, 1927) and the wave equation (Schrodinger, 1926).

\section{Definitions}

$\mathrm{m}_{0}=$ particle rest mass; for the electron $=9.109 \cdot 10^{-28} \mathrm{~g}$.

$\lambda_{\mathrm{C}}=$ Compton wavelength for a particle of specific rest mass $\left(\mathrm{m}_{0}\right)$; for the electron it is experimentally measured to be $=2.426 \cdot 10^{-10} \mathrm{~cm}$.

$\lambda_{\mathrm{B}}=$ deBroglie wavelength of a particle of specific rest mass at a known velocity.

$V_{\mathrm{p}}=$ velocity of particle.

$\mathrm{c}=$ velocity of light $=2.998 \cdot 10^{10} \mathrm{~cm} / \mathrm{s}$.

$\mathrm{h}=$ Planck's constant $=6.626 \cdot 10^{-27} \mathrm{~g} \cdot \mathrm{cm}^{2} / \mathrm{s}$.

$p=$ particle momentum $=\left[\mathrm{m}_{0} \cdot V_{\mathrm{p}}\right] \mathrm{g} \cdot \mathrm{cm} / \mathrm{s}$.

\section{Results}

\subsection{Relation to $\mathrm{c}$}

From the well-known deBroglie equation, $\lambda_{\mathrm{B}}=\mathrm{h} / p$, and with $p=m \cdot V_{\mathrm{p}}$, obtain the relation $\lambda_{\mathrm{B}} \cdot V_{\mathrm{p}}=\mathrm{h} / m$. Introduce the constant, $\kappa=\left[m \cdot \lambda_{\mathrm{C}}\right]_{\text {particle }}=2.21 \cdot 10^{-37} \mathrm{~g} \cdot \mathrm{cm}$, which holds for every particle. Then $\kappa \cdot \mathrm{c}=\mathrm{h}$ and $\lambda_{\mathrm{C}} \cdot \mathrm{c}=\mathrm{h} / m$, so for any particle this gives $\left[\left(\lambda_{\mathrm{B}} / \lambda_{\mathrm{C}}\right) \cdot V_{\mathrm{p}}\right]_{\text {particle }}=\mathrm{c}$.

This result for $\mathrm{c}$ is independent of particle mass. In the relation $\lambda_{\mathrm{B}}=\lambda_{\mathrm{C}} \cdot \mathrm{c} / V_{\mathrm{p}}$, when $V_{\mathrm{p}}$ approaches $\mathrm{c}$, then the magnitude of the matter-wave property of the particle, $\lambda_{B}$, decreases, more rapidly than $\lambda_{C}$ and approaches the magnitude of the equivalent $\lambda_{\mathrm{C}}$ for that particle. When $V_{\mathrm{p}}$ approaches zero the magnitude of $\lambda_{\mathrm{B}}$ increases and $\lambda_{\mathrm{B}} /$ $\lambda_{\mathrm{C}}$ approaches infinity, as expected. 
Introduce the new constant, $\beta_{\mathrm{p}}=\lambda_{\mathrm{B}} \cdot V_{\mathrm{p}}$, called here the deBroglie particle constant, for non-relativistic velocities where mass $=\mathrm{m}_{0}$, which varies from particle to particle because it depends on the particle's rest mass via $\lambda_{\mathrm{B}}$. A plot of $\lambda_{\mathrm{B}}$ versus $V_{\mathrm{p}}$ gives a rectangular hyperbola that is specific for each particle. Thus, $\lambda_{\mathrm{B}} \cdot V_{\mathrm{p}}=\lambda_{\mathrm{C}} \cdot \mathrm{c}=\beta_{\mathrm{p}}=\mathrm{h} /$ $\mathrm{m}_{0}$ offers different ways to calculate $\beta_{\mathrm{p}}$ for a particle of rest mass, $\mathrm{m}_{0}$. For the electron, $\beta_{\mathrm{e}}=\left(2.426 \cdot 10^{-10}\right.$ $\mathrm{cm}) \cdot\left(2.998 \cdot 10^{10} \mathrm{~cm} / \mathrm{s}\right)=7.27 \mathrm{~cm}^{2} / \mathrm{s}$. It gives for the proton, $\beta_{\mathrm{pr}}=\left(1.321 \cdot 10^{-13} \mathrm{~cm}\right) \cdot\left(2.998 \cdot 10^{10} \mathrm{~cm} / \mathrm{s}\right)=$ $3.96 \cdot 10^{-3} \mathrm{~cm}^{2} / \mathrm{s}$. The value of $\beta_{\mathrm{p}}$ depends inversely on the value of the particle's mass because a larger mass means a smaller $\lambda_{\mathrm{B}}$, as well as a smaller $\lambda_{\mathrm{C}}$. The values of all three parameters $\lambda_{\mathrm{B}}, V_{\mathrm{p}}$ and $\lambda_{\mathrm{C}}$ are accessible experimentally only for the electron.

At relativistic velocities $m$ is increasing as $V_{\mathrm{p}}$ increases, so $\beta_{\mathrm{p}}$ now becomes a variable instead of a constant. The value of $\lambda_{\mathrm{B}}$ depends on $\left\{\left[\left(\mathrm{c}^{2}\right)-\left(V_{\mathrm{p}}\right)^{2}\right] /\left(V_{\mathrm{p}}\right)^{2}\right\}^{1 / 2}$, whereas $\lambda_{\mathrm{C}}$ depends on $\left\{\left[\left(\mathrm{c}^{2}\right)-\left(V_{\mathrm{p}}\right)^{2}\right] / \mathrm{c}^{2}\right\}^{1 / 2}$. Thus, $\lambda_{\mathrm{B}}$ must decrease faster than $\lambda_{\mathrm{C}}$ when $V_{\mathrm{p}}$ is increasing. This satisfies $\lambda_{\mathrm{B}}=\lambda_{\mathrm{C}} \cdot \mathrm{c} / V_{\mathrm{p}}$.

\subsection{Uncertainty Relation}

The Uncertainty relation (Heisenberg, 1927, Wheeler \& Zurck, 1983) for pairs of non-commuting conjugate operators, such as position and momentum, is given by $(\Delta x \cdot \Delta p) \geq \mathrm{h} / 4 \pi$. It places a lower bound on the product of the standard deviations when considering measurements on non-commuting variables - particularly for preparation uncertain relations (Ma et al., 2016). The results obtained here offer another perspective on it. Rewriting in terms of $V_{\mathrm{p}}, \lambda_{\mathrm{B}}, \lambda_{\mathrm{C}}$ and $\beta_{\mathrm{p}}$ gives for any particle $\left(V_{\mathrm{p}}<<\mathrm{c}\right)$

$$
\Delta x \cdot \Delta p=\Delta x \cdot\left[\Delta\left(\mathrm{m}_{0} \cdot V_{\mathrm{p}}\right)\right] \geq \mathrm{m}_{0} \cdot\left(\lambda_{\mathrm{C}} \cdot \mathrm{c}\right) / 4 \pi=\mathrm{m}_{0} \cdot\left(\lambda_{\mathrm{B}} \cdot V_{\mathrm{p}}\right) / 4 \pi
$$

So, $\Delta x \cdot \Delta V_{\mathrm{p}} \geq\left(\beta_{\mathrm{p}} / 4 \pi\right) \mathrm{cm}^{2} / \mathrm{s}$ and then dividing by $\beta_{\mathrm{p}}$ yields the product of the dimensionless percentage errors, $\left(\Delta x / \lambda_{\mathrm{B}}\right) \cdot\left(\Delta V_{\mathrm{p}} / V_{\mathrm{p}}\right) \geq 1 / 4 \pi$.

For kinetic energy and time (assuming $\Delta t$ is seen as the time it takes for an observable to change by one standard deviation)

$$
\Delta t \cdot \Delta E_{\mathrm{kin}}=\Delta t \cdot\left(\mathrm{m}_{0} / 2\right) \cdot \Delta\left[\left(V_{\mathrm{p}}\right)^{2}\right] \geq \mathrm{m}_{0} \cdot\left(\lambda_{\mathrm{C}} \cdot \mathrm{c}\right) / 4 \pi=\mathrm{m}_{0} \cdot\left(\lambda_{\mathrm{B}} \cdot V_{\mathrm{p}}\right) / 4 \pi
$$

then $\Delta t \cdot \Delta\left[\left(V_{\mathrm{p}}\right)^{2}\right] \geq\left(\beta_{\mathrm{p}} / 2 \pi\right) \mathrm{cm}^{2} / \mathrm{s}$ for any particle. For the lower bound $(l b)$ case this leads to the relation $[\Delta x / \Delta t] \cdot\left[\Delta V_{\mathrm{p}} / \Delta\left(V_{\mathrm{p}}\right)^{2}\right]_{l b}=1 / 2$.

One other relevant pair of operators does not commute. It requires different units than $h$. The relation is then $\Delta x \cdot \Delta E_{\text {kin }}=\mathrm{cm} \cdot \mathrm{g} \cdot \mathrm{cm}^{2} / \mathrm{s}^{2}$, which leads to

$$
\Delta x \cdot \Delta\left[\left(V_{\mathrm{p}}\right)^{2}\right] \geq\left[\left(\beta_{\mathrm{p}} / 2 \pi\right) \cdot<V_{\mathrm{p}}>\right] \geq\left(\beta_{\mathrm{p}} / 2 \pi\right) \cdot V_{\mathrm{p}} \mathrm{cm}^{3} / \mathrm{s}^{2}
$$

Dividing by $\beta_{\mathrm{p}}$ yields the product of the dimensionless percentage errors given by the relation $\left(\Delta x / \lambda_{\mathrm{B}}\right) \cdot\left\{\Delta\left[\left(V_{\mathrm{p}}\right)^{2}\right] /\left(V_{\mathrm{p}}\right)^{2}\right\} \geq 1 / 2 \pi$.

All these Uncertainty relations are expressible in terms of the deBroglie particle constant introduced here, which is specific to each particle and therefore governs the magnitude of the Uncertainty for that particle.

\subsection{Wave Equation}

The deBoglie idea of a matter-wave that characterized a moving particle was established experimentally by electron diffraction on crystals (Davisson \& Germer, 1928) shortly after the serendipitous creation of the particle wave equation (Schrodinger, 1926). Given the concept of matter-waves, it seems reasonable to seek a differential equation (D.E.) that describes the relation between the relevant variables governing the behavior of the matterwave for a particle in motion, without assuming any empirical constants. This suggests a non-dimensional D.E. that employs the fractional change form, $\Delta_{\mathrm{f}}=d y / y$, the $\%$ change in the variable $y$. Define:

$d y / d t=$ velocity of $y$ with respect to $t$.

$d^{2} y / d t^{2}=$ acceleration of $y$ with respect to $t$.

$d y / d x=$ slope of the $y$ versus $x$ plot.

$(d / d x)(d y / d x)=$ gradient with respect to $x$ of the slope $=d^{2} y / d x^{2}=$ curvature.

Illustrations:

- Hooke's law for the position of the moving mass $\left(x_{\mathrm{m}}\right)$ on the end of the coiled spring is defined by the relation, the $\%$ change acceleration $=$ the $\%$ change distance, so $\Delta_{\mathrm{f}}\left(d^{2} x_{\mathrm{m}} / d t^{2}\right)=\Delta_{\mathrm{f}}\left(x_{\mathrm{m}}\right)$.

- Maxwell's second-order D.E. for $E$ is defined by the $\%$ change curvature $=$ the $\%$ change acceleration, so $\Delta_{\mathrm{f}}\left(\partial^{2} E / \partial x^{2}\right)=\Delta_{\mathrm{f}}\left(\partial^{2} E / \partial t^{2}\right)$. 
Such non-dimensional D.E. equations are free of assumptions about empirical constants. These emerge directly from the integration and consideration of the units.

Assume the matter-wave for a free particle (moving with fixed velocity, $V_{\mathrm{p}}<<\mathrm{c}$ ), in one dimension and not influenced by any external field) exhibits a periodic behavior. Assume a function $A(x, t)$ describes this behavior, where $A$ is related to the amplitude of the particle's matter-wave. For $A(x, t)$, the second-order partial D.E. follows from assuming that the behavior of $A(x, t)$ is governed by the following simple relation

$\%$ change curvature of $A=\%$ change velocity of $A$ giving

$$
\Delta_{\mathrm{f}}\left(\partial^{2} A / \partial x^{2}\right)=\Delta_{\mathrm{f}}(\partial A / \partial t)
$$

This defines the basic physical relationship for the variables governing the particle's matter-wave behavior under the given conditions. Integrating gives

$$
d^{2} A / d x^{2}=\mathrm{C}_{1} \cdot d A / d t
$$

where $\mathrm{C}_{1}$ has the units of $\mathrm{s} / \mathrm{cm}^{2}$. Thus, set $\mathrm{C}_{1}=\left(-i / \beta_{\mathrm{p}}\right)$ in terms of the deBroglie particle constant. Separating variables gives, $A(x, t)=\Psi(x) \Phi(t)$. Then for $\Phi(t)$,

$$
d \Phi(t) / \Phi(t)=\left(\mathrm{K} / \mathrm{C}_{1}\right) \cdot d t=-i \cdot \beta_{\mathrm{p}} /\left(\lambda_{\mathrm{B}}\right)^{2}
$$

where the separation constant, $\mathrm{K}$, has units of $\left(1 / \mathrm{cm}^{2}\right)$ giving $\mathrm{K}=\left(i / \lambda_{\mathrm{B}}\right)^{2}$, and so $\mathrm{K} / \mathrm{C}_{1}=\left(i / \lambda_{\mathrm{B}}\right)^{2} /\left(-i / \beta_{\mathrm{p}}\right)=-$ $i \cdot \mathrm{V}_{\mathrm{p}} / \lambda_{\mathrm{B}}$, for fixed velocity. After integrating,

$$
\Phi(t)=\mathrm{C}_{2} \cdot \exp \left[t \cdot \mathrm{K} / \mathrm{C}_{1}\right]=\mathrm{C}_{2} \cdot \exp \left[-i \cdot t \cdot \mathrm{V}_{\mathrm{p}} / \lambda_{\mathrm{B}}\right]
$$

The time-independent part of the equation has a solution,

$$
\Psi(x)=\mathrm{C}_{3} \cdot \exp \left[\mathrm{K}^{1 / 2} \cdot x\right]=\mathrm{C}_{3} \cdot \exp \left[i \cdot x / \lambda_{\mathrm{B}}\right]
$$

Then, with $\mathrm{C}_{2} \cdot \mathrm{C}_{3}=\mathrm{A}$,

$$
A(x, t)=\Psi(x) \Phi(t)=\mathrm{A} \cdot \exp \left[\left(i \cdot x-\mathrm{V}_{\mathrm{p}} \cdot t\right) / \lambda_{\mathrm{B}}\right]=\mathrm{A} \cdot \exp \left[i \cdot\left(x-x_{t}\right) / \lambda_{\mathrm{B}}\right]
$$

expressed in terms of $\lambda_{\mathrm{B}}$, the deBroglie wavelength of the particle's matter-wave, and $\mathrm{V}_{\mathrm{p}}$, its velocity, where $\left(\mathrm{V}_{\mathrm{p}} \cdot t\right)$ $=x_{t}$, the expected "classical" distance traveled. Thus, $\left(x-x_{t}\right)$ is the dispersion around this expected result, which is a direct consequence of the quantum mechanical nature of the wave function's prediction for the particle's location. This dispersion becomes a $\%$ change when expressed as $\left(x-x_{t}\right) / \lambda_{\mathrm{B}}$.

\section{Discussion}

The equation $\left[\lambda_{B} \cdot V_{\mathrm{p}}=\lambda_{\mathrm{C}} \cdot \mathrm{c}\right]_{\mathrm{particle}}=\beta_{\mathrm{p}}$ encapsulates a fundamental relation for the two matter-states, particle and wave. This result for $\mathrm{c}$ has not appeared previously in the literature. The analysis revealed the unique connection of $\mathrm{c}$ to $\lambda_{\mathrm{B}}, \lambda_{\mathrm{C}}$ and $V_{\mathrm{p}}$ via the new constants $\kappa$ and $\beta_{\mathrm{p}}$. Recognize $\kappa$ as the "mass-wave" constant for all particles and $\beta_{\mathrm{p}}$ as the matter-wave constant for any particular particle. It was essential to employ $\kappa \cdot \mathrm{c}=\mathrm{h}$ in order to obtain this result for $\mathrm{c}$.

The one-dimensional Schrodinger equation for the free non-relativistic electron was described in terms of $\lambda_{\mathrm{B}}$ and $V_{\mathrm{p}}$. This result reopens the question of what exactly is waving in the wave equation? It suggests $A(x, t)$ could be equated to the amplitude of the deBroglie matter-wave characterizing each solution of the wave function. The square of the amplitude of a classical wave is a measure of its relative intensity, which correlates with a relative probability. For relativistic velocities, $\lambda_{\mathrm{B}} \cdot V_{\mathrm{p}}=\lambda_{\mathrm{C}} \cdot \mathrm{c}=\beta_{\mathrm{p}}=\mathrm{h} / \mathrm{m}$, a variable.

Looking at $\mathrm{h}$ in terms of $\mathrm{h}=\mathrm{\kappa} \cdot \mathrm{c}=m \cdot \lambda_{\mathrm{C}} \cdot \mathrm{c}=m \cdot \lambda_{\mathrm{B}} \cdot V_{\mathrm{p}}=m \cdot \beta_{\mathrm{p}}$ reveals a different perspective on the Uncertainty principle, depending on the formulation of the specific variables involved. The role played by $h$ in the Uncertainty principle is also reconsidered in terms of the appropriate parameters, $\lambda_{\mathrm{B}}, \lambda_{\mathrm{C}}$ and $V_{\mathrm{p}}$ as well as $\beta_{\mathrm{p}}$ and $\kappa$. This gave new expressions for the standard forms of the Uncertainty relation that were particle specific. Thus, $\beta_{\mathrm{p}}=\mathrm{h} / \mathrm{m}=$ $\mathrm{h}_{\mathrm{p}}$ might also be interpreted as the "rationalized" Planck constant for any particle at velocity $V_{\mathrm{p}}$.

The equation $\left[\lambda_{B} \cdot V_{\mathrm{p}}=\lambda_{\mathrm{C}} \cdot \mathrm{c}\right]_{\text {particle }}$ shows the close relationship between the wave-like behavior of a particle, $\lambda_{B}$, and the electromagnetic wave equivalent of that particle's rest mass, $\lambda_{\mathrm{c}}$. As $V_{\mathrm{p}}$ approaches c, these wavelengths approach ever more closely. This suggests that the wave character of a particle might approximate the wave character of an electromagnetic wave in this case. 


\section{Conclusions}

The equation $\left[\lambda_{B} \cdot V_{\mathrm{p}}=\lambda_{\mathrm{C}} \cdot \mathrm{c}\right]_{\text {particle }}$ encapsulates a fundamental relation for the two matter-states, particle and wave. This result for $\mathrm{c}$ has not appeared previously in the literature. It revealed the unique connection of $\mathrm{c}$ to $\lambda_{\mathrm{B}}, \lambda_{\mathrm{C}}$ and $V_{\mathrm{p}}$ via the new constants $\kappa$ and $\beta_{\mathrm{p}}$. The utility of calculating $\beta_{\mathrm{p}}$ for the particle from $\lambda_{\mathrm{C}} \cdot \mathrm{c}=\lambda_{\mathrm{B}} \cdot V_{\mathrm{p}}$ was shown. Looking at $\mathrm{h}$ in terms of $\mathrm{h}=\kappa \cdot \mathrm{c}=m \cdot \lambda_{\mathrm{C}} \cdot \mathrm{c}=m \cdot \lambda_{\mathrm{B}} \cdot V_{\mathrm{p}}=m \cdot \beta_{\mathrm{p}}$ reveals a different perspective on the Uncertainty principle, depending on the formulation of the specific relations involved. The time-dependent wave equation for a free particle was derived in terms of $\lambda_{\mathrm{B}}$ and $V_{\mathrm{p}}$.

\section{References}

Compton, A. (1923). A quantum theory of the scattering of x-rays by light elements. Phys. Rev., 21, 483-502.

Davisson, C., \& Germer, L. (1928). Reflections of electrons by a crystal of Nickel. Proc. Nat. Acad. Scis., 14, $317-$ 332.

deBroglie, L. (1925). Recherches sur la theorie des quanta. Ann. de Phys., 3, 22.

Heisenberg, W. (1927). Ueber den anschaulichen Inhalt der quantentheoretischen kinematic and mechanic. Zeits. f. Physik., 44, 172-198.

Ma, W., Ma, Z., Wang H., Chen, Z., Liy, Y., Kong, F., ... Du, J. (2016). Experimental test of Heisenberg's uncertainty relation based on statistical distances. arXiv:1512.07407v2 (2016)

Schrodinger, E. (1926). Quantisierung als Eigenwortenproblem. Ann. d. Physik., 112, 361-376.

Wheeler, J., \& Zurck, W. (1983). Quantum Theory of Measurement. Princeton, NJ: Princeton University Press.

\section{Copyrights}

Copyright for this article is retained by the author(s), with first publication rights granted to the journal.

This is an open-access article distributed under the terms and conditions of the Creative Commons Attribution license (http://creativecommons.org/licenses/by/4.0/). 